\title{
Peroxisome proliferator-activated receptor $\delta$ agonist attenuates hepatic steatosis by anti-inflammatory mechanism
}

\author{
Mi Young Lee ${ }^{1}$, Ran Choi ${ }^{1}$, Hong Min Kim ${ }^{1}$, \\ Eun Ju Cho ${ }^{1}$, Bo Hwan Kim ${ }^{2}$, Yeon Sik Choi, \\ Jarinyaporn Naowaboot ${ }^{1}$, Eun Young Lee ${ }^{3}$, \\ Young Chul Yang ${ }^{4}$, Jang Yel Shin', \\ Young Goo Shin ${ }^{1}$ and Choon Hee Chung ${ }^{1,5,6}$

\section{${ }^{1}$ Department of Internal Medicine} \\ Yonsei University Wonju College of Medicine \\ Wonju 220-701, Korea \\ ${ }^{2}$ Department of Nursing \\ Gachon University of Medicine and Science \\ Incheon 406-799, Korea \\ ${ }^{3}$ Department of Internal Medicine \\ Soonchunhyang University College of Medicine \\ Cheonan 336-745, Korea \\ ${ }^{4}$ Department of Anatomy \\ ${ }^{5}$ Institute of Lifestyle Medicine \\ Yonsei University Wonju College of Medicine \\ Wonju 220-701, Korea \\ ${ }^{6}$ Corresponding author: Tel, 82-33-741-0506; \\ Fax, 82-33-731-5884; E-mail, cchung @yonsei.ac.kr \\ http://dx.doi.org/10.3858/emm.2012.44.10.066
}

Accepted 19 July 2012

Available Online 24 July 2012

Abbreviations: ALT, alanine transaminase; AST, aspartate transaminase; CPT-1, carnitine palmityl transferase-1; IPGTT, intraperitoneal glucose tolerance test; IVITT, intravenous insulin tolerance test; LETO, Long-Evans Tokushima Otsuka; MCP-1, monocyte chemo-attractant protein-1; NAFLD, non-alcoholic fatty liver disease; $\mathrm{NASH}$, nonalcoholic steatohepatitis; OLETF, Otsuka Long-Evans Tokushima Fatty; PGC-1 $\alpha$, peroxisome proliferator-activated receptor- $\gamma$ coactivator-1 $\alpha$; PPAR, peroxisome proliferator-activated receptors; PPRE, peroxisome proliferators response element; RXR, retinoid X-receptor; TG, triglyceride

\begin{abstract}
Although peroxisome proliferator receptor (PPAR)- $\alpha$ and PPAR- $\gamma$ agonist have been developed as chemical tools to uncover biological roles for the PPARs such as lipid and carbohydrate metabolism, PPAR- $\delta$ has not been fully investigated. In this study, we examined
\end{abstract}

\begin{abstract}
the effects of the PPAR- $\delta$ agonist GW0742 on fatty liver changes and inflammatory markers. We investigated the effects of PPAR- $\delta$ agonist GW0742 on fatty liver changes in OLETF rats. Intrahepatic triglyceride contents and expression of inflammatory cytokines such as tumor necrosis factor- $\alpha$ (TNF- $\alpha$ ) and monocyte chemo-attractant protein-1 (MCP-1) and also, PPAR- $\gamma$ coactivator (PGC)-1 $\alpha$ gene were evaluated in liver tissues of OLETF rats and HepG2 cells after GW0742 treatment. The level of TNF- $\alpha$ and MCP-1 was also examined in supernatant of Raw264. 7 cell culture. To address the effects of GW0742 on insulin signaling, we performed in vitro study with AML12 mouse hepatocytes. Rats treated with GW0742 (10 mg/kg/day) from 26 to 36 weeks showed improvement in fatty infiltration of the liver. In liver tissues, mRNA expressions of TNF- $\alpha$, MCP-1, and PGC- $1 \alpha$ were significantly decreased in diabetic rats treated with GW0742 compared to diabetic control rats. We also observed that GW0742 had inhibitory effects on palmitic acid-induced fatty accumulation and inflammatory markers in HepG2 and Raw264.7 cells. The expression level of Akt and IRS-1 was significantly increased by treatment with GW0742. The PPAR- $\delta$ agonist may attenuate hepatic fat accumulation through anti-inflammatory mechanism, reducing hepatic PGC-1 $\alpha$ gene expression, and improvement of insulin signaling.
\end{abstract}

Keywords: diabetes mellitus; fatty liver; GW0742; inflammation; monocyte chemo-attractant protein-1; PPAR $\delta$; PPARGC1A protein, human; tumor necrosis factor- $\alpha$

\section{Introduction}

Peroxisome proliferator-activated receptors (PPARs) heterodimerized with the retinoid X-receptor (RXR), one of the nuclear receptors, have been known to be involved in lipid and carbohydrate metabolism after binding to peroxisome proliferators response element (PPRE) (Kliewer et al., 1999). Currently, three genes (PPAR- $\alpha$, PPAR- $\gamma$, and PPAR- $\delta$ ) in the PPAR family are known. PPAR- $\alpha$ is most abundantly 
expressed in the liver and has the effect of diminishing circulating triglycerides and increasing high dense lipoprotein (HDL) cholesterol. PPAR- $\gamma$ is present in high concentrations in adipose tissue and improves insulin sensitivity. These effects are well documented, but the function of PPAR- $\delta$ has not been fully investigated (Berger et al., 2005).

Many studies have reported that low-grade chronic systemic inflammatory response to nutrient excess is related to obesity, insulin resistance, type 2 diabetes mellitus, and non-alcoholic fatty liver disease (NAFLD), which refers to a wide spectrum of liver disease ranging from simple fatty liver (steatosis), to nonalcoholic steatohepatitis (NASH), and cirrhosis (Wellen and Hotamisligil, 2005; Shoelson et al., 2007; Tarantino et al., 2010). It is reported that hepatic steatosis, inflammation, and apoptosis are induced by neutrophil infiltration and the production of pro-inflammatory cytokines associated with excessive free fatty acid released from adipocytes (Lawson et al., 1998).

Recent studies showed that a PPAR- $\delta$ agonist could prevent lipopolysaccharide (LPS)-induced expression and secretion of pro-inflammatory cytokines in 3T3-L1 adipocytes and reduce atherosclerosis through anti-inflammatory effects in apoE knockout mice (Barish et al., 2008; Rodriguez-Calvo et al., 2008). However, as of yet, it is not known if PPAR- $\delta$ agonist influence NAFLD through anti-inflammatory mechanism.

In this study, we investigated whether the PPAR- $\delta$ agonist GW0742 could improve fatty liver changes by decreasing inflammatory cytokines in a type 2 diabetic rat model (for in vivo study) and in HepG2 and Raw264.7 cells (for in vitro study). We also examined whether PPAR- $\delta$ agonist alters the expression of genes related to fat accumulation in the liver such as peroxisome proliferator-activated receptor (PPAR)- $\gamma$ coactivator (PGC)- $1 \alpha$ and carnitine palmityl transferase-1 (CPT-1) and insulin signaling pathway.

\section{Results}

\section{Anthropometric and biochemical characteristics of experimental rats}

The anthropometric and biochemical data of experimental rats are shown in Table 1. Compared to the control LETO group, the body weight of diabetic control (DM) and GW0742-treated diabetic (DM + GW) groups was significantly increased over the experimental period. Although no difference in body weight was observed between the diabetic control and GW0742 treated diabetic rat group at the end of the study, the amount gained in body weight was significantly lower in the GW0742-treated diabetic rat group compared to the diabetic control group. Epididymal fat weight on the left and right sides of the control group were significantly lower than the diabetic rat groups. Epididymal fat weights of the GW0742-treated diabetic rat group were significantly lower than the diabetic control group. The total cholesterol level was significantly increased in only the diabetic control group compared to the normal control group. However, triglyceride levels were significantly increased in both the diabetic control and GW0742-treated diabetic rat groups compared to the normal control group. No differences

Table 1. Anthropometric and biochemical characteristics of experimental rats

\begin{tabular}{lccc}
\hline & Control & DM & DM + GW \\
\hline Initial body weights (g) & $482.00 \pm 31.80$ & $562.44 \pm 32.84^{*}$ & $589.00 \pm 49.7^{*}$ \\
Final body weights (g) & $518.11 \pm 35.83$ & $627.00 \pm 9.02^{*}$ & $616.37 \pm 58.57^{*}$ \\
Weight change & $36.11 \pm 10.50$ & $64.55 \pm 24.30^{*}$ & $26.62 \pm 25.02^{\dagger}$ \\
Epididymal fat pad, left (g) & $5.11 \pm 0.96$ & $8.66 \pm 0.75^{*}$ & $7.22 \pm 1.20^{*, \dagger}$ \\
Epididymal fat pad, right (g) & $5.05 \pm 1.37$ & $8.22 \pm 0.87^{*}$ & $6.44 \pm 1.44^{*},^{\dagger}$ \\
Total cholesterol (mg/dl) & $70.33 \pm 8.86$ & $92.22 \pm 22.40^{*}$ & $86.33 \pm 27.52$ \\
Triglyceride (mg/dl) & $23.33 \pm 10.36$ & $189.22 \pm 86.81^{*}$ & $225.88 \pm 132.60^{*}$ \\
HDL cholesterol (mg/dl) & $58.11 \pm 6.03$ & $67.66 \pm 13.66$ & $63.44 \pm 15.58$ \\
AST (IU/L) & $56.55 \pm 6.30$ & $50.11 \pm 15.71$ & $47.55 \pm 13.48$ \\
ALT (IU/L) & $25.00 \pm 2.95$ & $28.66 \pm 6.87$ & $39.55 \pm 24.54^{*}$ \\
Adiponectin ( $\mu \mathrm{gg} / \mathrm{ml})$ & $5.29 \pm 0.43$ & $5.6 \pm 0.90$ & $5.39 \pm 0.97$ \\
MCP-1 (pg/ml) & $82.19 \pm 32.25$ & $91.74 \pm 34.11$ & $90.68 \pm 24.60$ \\
Kitt & $4.13 \pm 0.83$ & $1.87 \pm 1.45^{*}$ & $3.78 \pm 1.31^{*, \dagger}$ \\
\hline
\end{tabular}

Data are mean \pm S.D. Control, normal control group; DM, diabetic control group; DM + GW, GW0742 treated diabetic rat group; HDL, high density lipoprotein; AST, aspartate transaminase; ALT, alanine transaminase; MCP-1, monocyte chemoattractant protein-1; Kitt, rate constant for glucose disappearance in the insulin tolerance test.

${ }^{*} P<0.05$ vs. Control. ${ }^{\dagger} P<0.05$ vs. DM. 

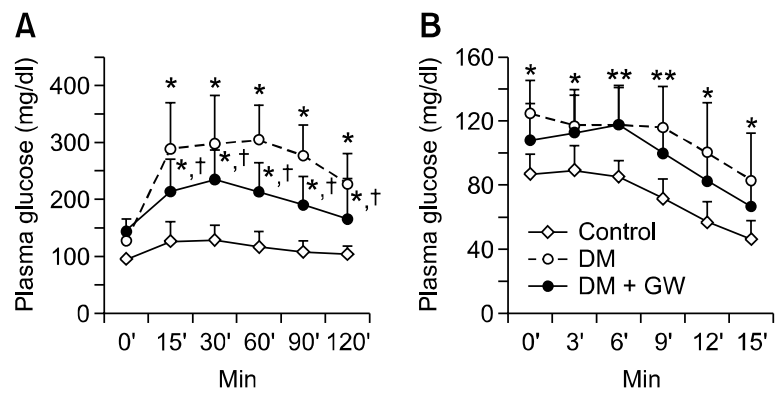

Figure 1. Changes in plasma glucose during IPGTT and IVITT. Plasma glucose responses in the IPGTT (A) and IVITT (B) in the normal control group (Control, $n=9$ ), diabetic control group (DM, $n=9$ ), and GW0742 treated diabetic rat group (DM $+\mathrm{GW}, n=9$ ) at the end of the study. ${ }^{*} P$ $<0.05$ vs control; $P<0.05$ vs DM.
A

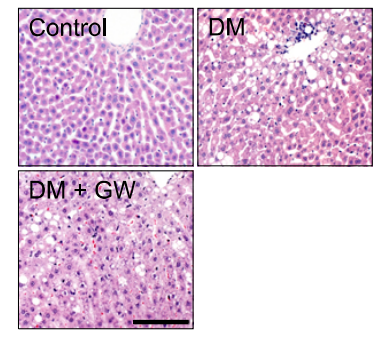

B

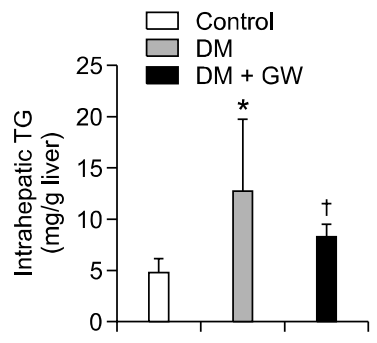

Figure 2. Histological findings of liver and intrahepatic TG content. (A) Representative images of H\&E staining were shown. In the diabetic control group, macrovesicular steatosis occupied greater than normal control group. GW0742 treated diabetic rat group showed markedly decreased fatty accumulation compared to the diabetic control group. (B) Analysis of intrahepatic TG was shown. The increased intrahepatic TG level of the diabetic control group was significantly decreased by GW0742 treatment. TG, triglyceride; Control, normal control group; DM, diabetic control group; DM + GW, GW0742 treated diabetic rat group. ${ }^{*} P<0.05$ vs control; ${ }^{\dagger} P<0.05$ vs DM. $\times 200$; scale bar, $50 \mu \mathrm{m}$.

were observed in the serum levels of adiponectin or MCP-1 among the groups.

\section{Intraperitoneal glucose tolerance test and intravenous insulin tolerance test}

Changes in plasma glucose during IPGTT in each experimental group at 35 weeks of age are illustrated in Figure 1A. Plasma glucose level at each time point of $15,30,60,90$, and 120 min was significantly decreased in the GW0742-treated diabetic group compared to the diabetic control group. Plasma glucose levels during IVITT in each experimental group are shown in Figure 1B and measured rate constant for plasma glucose disappearance (Kitt) are shown in Table 1. The plasma glucose level of diabetic control group was significantly increased during IVITT periods compared to the normal control group, and the
A

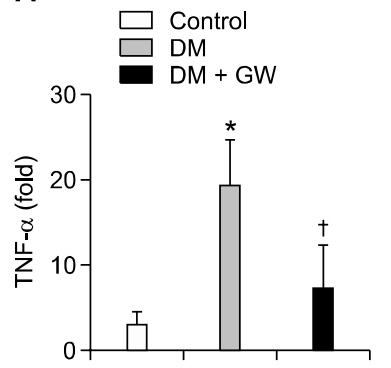

B

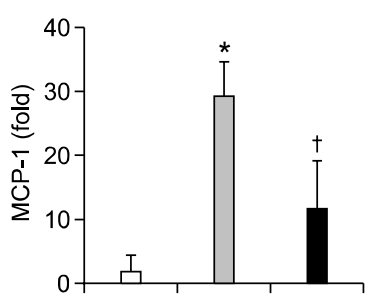

C
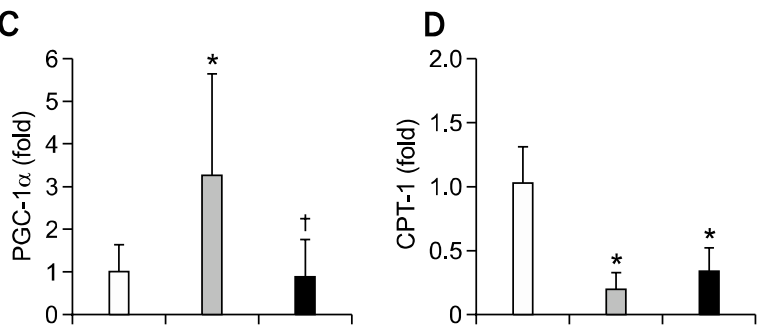

Figure 3. Relative mRNA expression of TNF- $\alpha$ (A), MCP-1 (B), PGC-1 $\alpha$ $(C)$, and CPT-1 (D) in liver tissues. Inflammatory cytokine expression was significantly decreased in GW0742 treated diabetic rat group (DM + GW) compared to the diabetic control group (DM). The mRNA expression of PGC-1 $\alpha$ was significantly decreased by GW0742 treatment. The mRNA expression of CPT-1 was significantly decreased in both DM and DM + GW groups compared with that of normal control group. ${ }^{*} P<0.05$ vs control; ${ }^{\dagger} P<0.05$ vs DM.

glucose level of the GW0742-treated diabetic rat group was significantly increased to those of diabetic control group at 6 and 9 min after insulin injection during IVITT. Overall, the Kitt value of GW0742-treated diabetic rat group was significantly decreased compared to the diabetic control group.

\section{Histological finding and intrahepatic lipid levels of liver}

All liver tissues were analyzed with $\mathrm{H} \& \mathrm{E}$ staining (Figure 2A). Elevated accumulation of fatty droplet was observed in both diabetic rat groups compared to the normal control group. However, GW0742-treated diabetic rat group showed decreased fatty accumulation compared to the diabetic control group. The triglyceride level of liver tissues obtained from diabetic control rat group was significantly higher than the normal control group. However, compared to the diabetic control group, GW0742treated diabetic rat group had significantly lower intrahepatic TG level (Figure 2B). 
A

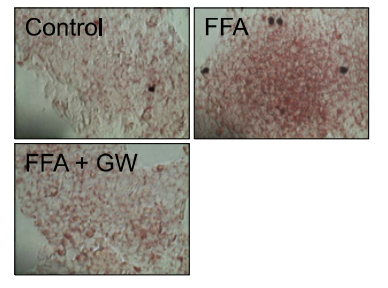

C

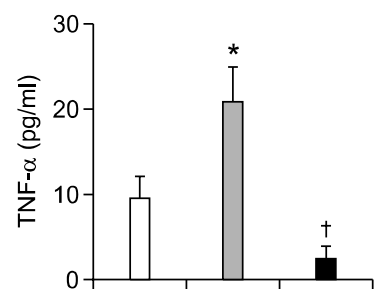

B

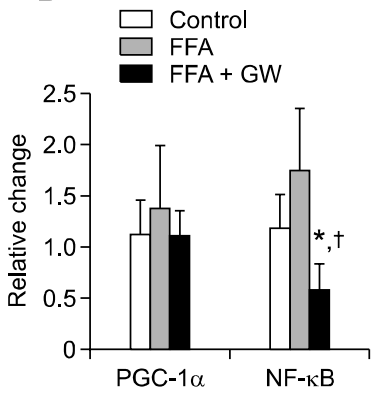

D

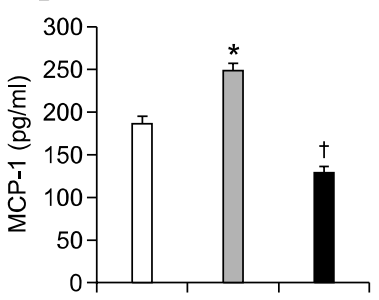

Figure 4. The data of HepG2 and RAW 264.7 cells. HepG2 cells were cultured with DMSO, $300 \mu \mathrm{M}$ palmitic acid and co-administration of 300 $\mu \mathrm{M}$ palmitic acid and $10 \mu \mathrm{M}$ of GW0742. (A) Cells were stained with Oil-red-O to measure intracellular lipid accumulation. Intracellular lipid vacuoles were markedly reduced by GW0742 treatment. (B) The mRNA expression levels of TNF- $\alpha, N F-\kappa B$ and PGC- $1 \alpha$ in HepG2 cells were shown. Cells treated with GW0742 showed significant reduction of $N F-\kappa B$ expression and a tendency to have decreased mRNA for TNF- $\alpha$ and PGC-1 $\alpha$. In study of RAW 264.7 cells, the secretion of TNF- $\alpha$ and MCP-1 was significantly increased after palmitic acid treatment compared with control group and the increased level of TNF- $\alpha$ and MCP-1 in co-administration of palmitic acid and GW 0742 compound group was significantly decreased ( $C$ and $D)$. FFA, free fatty acid (palmitic acid); TNF- $\alpha$, tumor necrosis factor- $\alpha$; NF- $\kappa B$, nuclear factor- $\kappa B$; MCP-1, monocyte chemoattractant protein-1; PGC-1 $\alpha$, peroxisome proliferator activated receptor (PPAR)- $\gamma$ coactivator- $1 \alpha$. ${ }^{*} P<0.05$ vs control; ${ }^{\dagger} P<$ 0.05 vs FFA. $\times 400$; scale bar, $127 \mu \mathrm{m}$.

\section{Real-time RT-PCR for TNF- $\alpha$, MCP-1, PGC- $1 \alpha$, and CPT-1 in rat liver tissues}

To determine the anti-inflammatory effects of GW0742 in the liver, we used real-time RT-PCR to examine TNF- $\alpha$ and MCP-1 mRNA, which are representative inflammatory cytokines, in experimental rat liver tissues. Quantitative analysis revealed that TNF- $\alpha$ and MCP-1 mRNA levels were significantly increased in the diabetic control group compared to the normal control group, and GW0742-treated diabetic rat group showed significantly decreased TNF- $\alpha$ and MCP- 1 mRNA compared to the diabetic control group (Figures $3 \mathrm{~A}$ and $3 \mathrm{~B}$ ). To investigate whether GW0742 altered gene expression related to fat accumulation in liver, we examined the mRNA levels of PGC- $1 \alpha$ and CPT-1. PGC- $1 \alpha$ mRNA in the diabetic control group showed an approximately three-fold increase compared to the normal control group, which was significantly decreased in

A

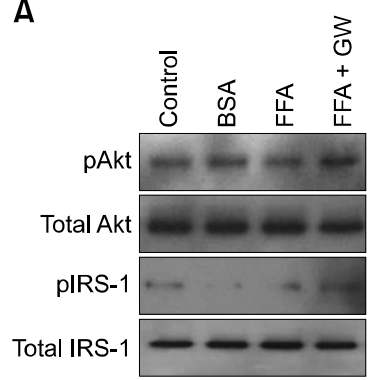

B

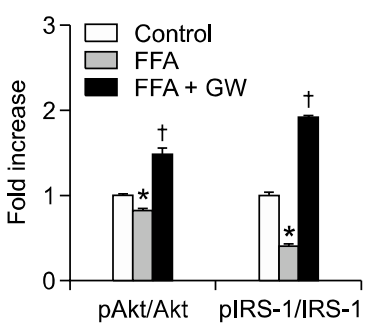

Figure 5. The data of AML12 cell. (A) Representative bands of western blot for pAkt, total Akt, pIRS-1, and total IRS-1 were shown. (B) Western blot analysis showed significantly decreased level of pAkt/Akt and pIRS-1/IRS-1 with treatment of FFA only and the decreased level of pAkt/Akt and pIRS-1//RS-1 was significantly increased with co-administration of FFA and GW treated group. ${ }^{*} P<0.05$ vs control; ${ }^{\dagger} P<0.05$ vs FFA.

GW0742-treated diabetic rat group (Figure 3C). In contrast, the mRNA of CPT-1 was significantly decreased in both diabetic rat groups compared to the normal control group. CPT-1 mRNA level was not different between diabetic control and GW0742-treated diabetic rat groups (Figure 3D).

\section{In vitro treatment of HepG2 and RAW 264.7 cells with GW0742}

Human HepG2 cells were used to extend the results from OLETF rats to a cellular model. To induce lipid accumulation in HepG2 cells, cells were exposed to a pathophysiologically relevant concentration of the plasma free fatty acid (palmitic acid) and $10 \mu \mathrm{M}$ of GW0742. Intracellular lipid vacuoles induced by palmitic acid were confirmed by Oil-red-O staining (Figure 4A). The increase in lipid vacuoles in HepG2 cells induced by palmitic acid was markedly diminished by co-administration of $10 \mu \mathrm{M}$ of GW0742 (Figure 4A). The expression levels of nuclear factor- $\mathrm{KB}(\mathrm{NF}-\mathrm{kB})$ and PGC-1 $\alpha$ were slightly increased in palmitic acid-treated HepG2 cells compared to the control group. This increase expression of PGC-1 $\alpha$ mRNA was slightly attenuated by GW0742 treatment, but these differences were not significant. On the other hand, cells treated with GW0742 showed significant reduction of NF-KB expression (Figure 4B).

To observe the changes of inflammatory cytokines in more detail, we utilized macrophage cell lines. The secretion of TNF- $\alpha$ and MCP-1 was significantly increased after palmitic acid treatment compared with control group: co-administration of palmitic acid and GW0742 compound group significantly attenuated the elevation of the cytokines (Figures $4 C$ and $4 D$ ). 


\section{In vitro assessment of AML12 cell with GW0742}

To address the effects of GW0742 on insulin signaling, we studied the pAkt and pIRS-1 expression in AML12 cells. Western blot analysis found the expression level of pAkt/Akt and pIRS-1/IRS-1 to be significantly decreased in palmitic acid-treated group. This decreased level of pAkt/Akt and pIRS-1/IRS-1 was improved with treatment by GW0742 (Figure 5).

\section{Discussion}

In this study, we found a protective function of PPAR- $\delta$ agonist GW0742 on hepatic steatosis. This protective role was ascertained in both type 2 diabetic rat model and HepG2 cells.

Among the PPAR subtypes, PPAR- $\gamma$ and PPAR- $\alpha$ are already targets for the treatment of type 2 diabetes and dyslipidemia (Kahri et al., 1993; Nolan et al., 1994; Miyazaki et al., 2001). On the other hand, the therapeutic potential of PPAR- $\delta$ on insulin resistance and fatty acid metabolism is still under investigation. The known key features of PPAR- $\delta$ activation are increase in fatty acid oxidation in skeletal muscle and cholesterol flux from peripheral tissues (Oliver et al., 2001; Tanaka et al., 2003; Evans et al., 2004). Recently, PPAR- $\delta$ agonist was reported to reduce inflammation in adipose tissue or vascular smooth muscle cells (Barish et al., 2008; Rodriguez-Calvo et al., 2008). Because inflammatory process was known to participate in insulin resistance, type 2 diabetes, and cardiovascular disease (Wellen and Hotamisligil, 2005; Shoelson et al., 2007; Jeong et al., 2011), inhibition of inflammation might be one targets of intervention in overcoming insulin resistance. Recent studies demonstrated that PPAR- $\delta$ agonist could inhibit lipopolysaccharide (LPS)-induced expression of MCP-1 and IL-6 in 3T3-L1 adipocytes and TNF- $\alpha$-induced NF- $\kappa B$ activation in human umbilical vein endothelial cells (HUVECs) (Fan et al., 2008; Rodriguez-Calvo et al., 2008). To confirm the anti-inflammatory effect of PPAR- $\delta$ agonist, we investigated MCP-1 expression level, which is increased in inflammatory and insulin resistant states, in 3T3-L1 adipocytes. We observed the induction of MCP-1 expression mediated by TNF- $\alpha$ in 3T3-L1 adipocytes, which PPAR- $\delta$ agonist significantly suppressed (data not shown). This finding is consistent with previous studies (Fasshauer et al., 2004) that reported reduced cytokine expression levels in the presence of a PPAR- $\delta$ agonist. In addition, we found that treatment with PPAR- $\delta$ agonist could decrease plasma glucose levels and improve Kitt, a marker of insulin sensitivity. Although PPAR- $\delta$ agonist treatment did not reduce body weights of OLETF rats, PPAR- $\delta$ agonist-treated OLETF rats gained significantly less weight than diabetic control group had significantly decreased epididymal fat weights. . These findings indirectly implicated the role of PPAR- $\delta$ agonist on insulin resistance.

In patients with metabolic syndrome, the prevalence of non-alcoholic fatty liver disease (NAFLD) is twoor three time higher than in general population (Marchesini et al., 2003). Currently, there is no approved therapy for NAFLD, and identifying research area for effective treatment of NAFLD has been mostly unsuccessful. NAFLD refers to a wide spectrum of liver disease ranging from simple fatty liver (steatosis), to nonalcoholic steatohepatitis (NASH) and cirrhosis. The pathologic findings of $\mathrm{NASH}$ are characterized by microvesicular or macrovesicular steatosis, inflammation, hepatocyte degeneration, and sometimes fibrosis (Bugianesi et al., 2005). In particular, inflammatory processes secondary to insulin resistance are regarded as a characteristic finding of NASH (McCullough, 2006). To investigate the effects of PPAR- $\delta$ on hepatic steatosis, we performed a series of experiments on a type 2 diabetic rat model and on HepG2 cells. Significant differences in liver histology were seen between diabetic control rats and PPAR- $\delta$ agonist-treated rats. In addition, lipid vacuoles were significantly decreased in HepG2 cells cultured with combination of palmitic acid and PPAR- $\delta$ agonist compared to cells treated with palmitic acid only. Because palmitic acid is known to induce a hyperlipidemic condition via inflammatory liver injury (Joshi-Barve et al., 2007; Gao et al., 2010), it could be thought that palmitic acid treatment sufficiently caused hepatic steatosis and inflammation. These findings may result from a reduction in liver tissue inflammation, as confirmed by RT-PCR. However, treatment with PPAR- $\delta$ agonist did not attenuate TNF- $\alpha$ or MCP-1 in HepG2 cells, which was expected. The discrepancy between rat liver tissue and in vitro cell lines may have been due to the source of inflammatory cytokines: the immune cells may release the cytokines in greater quantity than hepatocytes. In this experiment, significant attenuation of inflammatory response other than decreased $\mathrm{NF}-\kappa \mathrm{B}$ was not observed after treating HepG2 cells with GW0742. In contrast, macrophage cell lines demonstrated attenuation of both TNF- $\alpha$ and MCP-1 with treatment by GW0742. These findings suggest that the effect of inflammatory cytokines released by hepatocytes is relatively small compared to intrahepatic macrophage infiltration and the resulting inflammatory response caused by insulin resistance, and that PPAR- $\delta$ agonist attenuates the inflammatory response to improve hepatic steatosis. 
Confirming the macrophage infiltration within the liver tissue may have been necessary for a more accurate assessment.

In addition to these experiments, the expression of Akt and IRS-1 after treatment with GW0742 was investigated to observe whether PPAR- $\delta$ agonist directly acts on insulin signaling and improves insulin resistance. Western blot analysis was performed to assess the expression of Akt and IRS-1 in AML12 cells treated with GW0742: the expression of both signaling molecules was increased, implying that PPAR- $\delta$ agonist does directly improve insulin resistance at the liver and therefore, improves fatty liver.

One limitation of this experiment includes neglecting the Kupffer cells. Kupffer cells are macrophages residing in the liver and are known to be activated once the liver is injured by various agents, including fat deposition. Activated Kupffer cells are known to release a wide array of inflammatory cytokines and chemokines, which in turn may influence the phenotype of the surrounding cells, such as hepatocytes, stellate cells, and other immune cells (Bataller et al., 2005). Therefore, whether the apparent amelioration of the inflammation of the liver by PPAR- $\delta$ agonist is due to altered condition of Kupffer cells rather than due to infiltration of macrophages recruited from circulation should have been investigated.

The anti-inflammatory effects of the PPAR- $\delta$ agonist on the liver were important, and could be of clinical interest because few effective treatment strategies exist for NAFLD. Although the mechanism for the beneficial effect of PPAR- $\delta$ on the liver is partially explained by similar anti-inflammatory activity in adipose tissues and vascular smooth muscle cells, the therapeutic effects on NAFLD could be, in part, mediated through expression of other hepatic genes. Therefore, we examined the genes known to induce fatty liver. Through investigation of various genes, we found that PPAR- $\delta$ agonist decreased the expression of PGC-1 $\alpha$. PGC-1 $\alpha$ is a transcriptional co-activator involved in thermogenesis and energy metabolism (Liang and Ward, 2006). It is well elucidated that PGC- $1 \alpha$ could promote fatty acid oxidation, reduce fat accumulation, increase GLUT4 expression in muscle, and eventually lead to improvement of insulin resistance (Michael et al., 2001; Lin et al., 2002). However, PGC-1 $\alpha$ expression is reported to be elevated in type 2 diabetic mice, and elevated hepatic PGC- $1 \alpha$ expression was reported to increase PEPCK and contribute to fatty liver (Koo et al., 2004). Thus, the regulation of hepatic PGC-1 $\alpha$ expression is considered to be another process of fatty liver changes, along with inflammation. In our study, treatment with PPAR- $\delta$ agonist significantly reduced PGC-1 $\alpha$ expression in the liver tissue. Furthermore, although the current in vitro results may not show significant differences, potential evidence was seen for reduction of PGC-1 $\alpha$. However, the paradoxical actions of PGC-1 $\alpha$ in the muscle and the liver are controversial, and therefore, the effects of PPAR- $\delta$ agonist on hepatic PGC-1 $\alpha$ expression warrants further study. In addition, examining the effects of the PPAR- $\delta$ agonist on different tissues would be beneficial.

Patients with type 2 diabetes are at a higher risk of NAFLD and other inflammatory processes. According to our results, PPAR- $\delta$ agonist could be involved in glycemic control and positively influence fatty liver changes in type 2 diabetes and in insulinresistant state.

In summary, the PPAR- $\delta$ agonist GW0742 attenuated hyperglycemia and fatty accumulation in the liver. These changes were possibly due to suppression of inflammatory cytokines, such as TNF- $\alpha$ and MCP-1. The beneficial effect on hepatic fatty accumulation was also partially explained by the reduction in PGC-1 $\alpha$ expression and improvement in insulin signaling. Our findings suggest that PPAR- $\delta$ agonist has beneficial effects against NAFLD.

\section{Methods}

\section{Materials}

The synthetic PPAR- $\delta$ agonist GW0742 was kindly provided by GlaxoSmithKline (Brentford, United Kingdom). Palmitic acid and other chemicals were purchased from Sigma Aldrich (St. Louis, MO). All animal procedures were approved by the Institutional Animal Care and Use Committee (IACUC), Yonsei University at Wonju Campus. At 25 weeks, 18 male Otsuka-Long-Evans-Tokushima-Fatty (OLETF, Otsuka Pharmaceutical, Tokushima, Japan) and 9 Long-Evans-Tokushima-Otsuka (LETO) rats were divided into three groups: diabetic control group, GW0742 treated diabetic rat group, and normal control group. Animals were housed at constant temperature $\left(20-22^{\circ} \mathrm{C}\right)$ and humidity (50-60\%) with a 12-h light and 12-h dark cycle. They had free access to water and standard rat chow until 36 weeks of age. The experimental group received GW0742 (10 $\mathrm{mg} / \mathrm{kg} /$ day) by oral gavage tube from 26 weeks to 36 weeks. At 25 and 35 weeks, body weight, intraperitoneal glucose tolerance test (IPGTT), and intravenous insulin tolerance test (IVITT) were measured and a 24-h urine sample was collected. Plasma glucose disposal rate (Kitt; $\% / m i n)$, which indicates the time necessary to reduce the basal glucose level by one-half, was calculated as $0.693 / t 1 / 2$, where $t 1 / 2$ was determined from the slope of the glycemic concentrations from 3 to 15 min after intravenous regular insulin injection $(0.1 \mathrm{U} / \mathrm{kg})$. At 36 weeks, experimental rats were anesthetized with Zoletil ${ }^{\circledR}$ (Virbac Laboratories, Carros, France) by intraperitoneal injection and liver tissues were extracted. A portion of the liver tis- 
sue sample was frozen using liquid nitrogen and kept at $-70^{\circ} \mathrm{C}$ for mRNA and protein analysis. Another portion was fixed in $4 \%$ paraformaldehyde for $48 \mathrm{~h}$ and embedded in paraffin for histological examination.

\section{Cell cultures}

HepG2 cells obtained from Korean Cell Line Bank (KCLB, Seoul, Korea) were cultured in minimum Eagle's medium (Invitrogen Life Technologies Inc. , Carlsbad, CA) supplemented with $10 \%$ FBS (HyClone Laboratories Inc, Logan, UT), 100 units $/ \mathrm{ml}$ penicillin (Invitrogen), and $0.1 \mathrm{mg} / \mathrm{ml}$ streptomycin (Invitrogen). Cells were maintained at $37^{\circ} \mathrm{C}$ with humidified air and $\mathrm{CO}_{2}(5 \%)$. After $24 \mathrm{~h}, 10 \mu \mathrm{M}$ of GW0742 dissolved in dimethylsufoxide (DMSO) (SigmaAldrich) or DMSO was added to the medium. After $2 \mathrm{~h}$, palmitic acid $(300 \mu \mathrm{M})$ was added to the medium and cells were cultured for an additional $24 \mathrm{~h}$ before lysis and purification of total RNA. Subconfluent monolayers of HepG2 cells were stained with Oil-Red-O to determine fat accumulation. Dishes were washed with cold phosphate-buffered saline and fixed in 10\% paraformaldehyde for $5 \mathrm{~min}$. Oil-Red-O was added with agitation for $15 \mathrm{~min}$, followed by washing in $60 \%$ isopropanol. Cells were rinsed in distilled water and examined by light microscopy.

RAW264.7 macrophages (ATCC, Manassas, VA) was grown at $37^{\circ} \mathrm{C}$ in $5 \% \mathrm{CO}_{2}$ using Dulbecco's Modified Eagle's Medium (HyClone) containing $10 \%$ fetal bovine serum (FBS), $10 \mathrm{ml} / \mathrm{L}$ penicillin streptomycin (Invitrogen).

AML12 mouse hepatocytes (ATCC) were cultured in DMEM/F-12 media (HyClone) supplemented with 10\% FBS, antibiotics (100 units $/ \mathrm{ml}$ penicillin and $100 \mathrm{mg} / \mathrm{ml}$ streptomycin), $0.1 \mathrm{mM}$ dexamethasone, and a mixture of insulin, transferrin, and selenium (Invitrogen). RAW264.7 and AML12 were pretreated for $4 \mathrm{~h}$ with $10 \mu \mathrm{M}$ GW0742. For the induction of hepatocyte steatosis, subsets of RAW264.7 macrophages and AML12 hepatocytes were treated with palmitic acid ( $250 \mu \mathrm{M}$; Sigma-Aldrich) for $16 \mathrm{~h}$.

\section{Measurement of cytokines by ELISA}

Cytokines in culture supernatants and tissue homogenates of RAW264.7 macrophages were assayed by enzymelinked immunosorbent assays (ELISA). The assays were conducted using OptEIA mouse TNF- $\alpha$, a mouse MCP-1 set (BD Bioscience Pharmingen, $C A$ ). Cytokine levels were quantified from standard curves using the BioTek curvefitting program (BioTek, winooski).

\section{Western blot analysis}

Samples of $20-50 \mu \mathrm{g}$ total protein from AML12 mouse hepatocytes were subjected to Western blot analysis using polyclonal antibodies to phosphorylated Akt (Akt-pSer ${ }^{473}$ ), total Akt, phosphorylated insulin receptor substrate-1 $\left(\right.$ IRS1-pSer ${ }^{308}$ ), and total insulin receptor substrate-1 (Cell Signaling, Beverly, MA).

\section{Histologic examination of liver and serum chemistry}

Paraffin-embedded 5- $\mu \mathrm{m}$ liver tissue slices were stained with hematoxylin and eosin (H\&E). Blood samples were taken by cardiac puncture, and serum levels of total cholesterol, triglyceride (TG), HDL cholesterol, aspartate transaminase (AST), and alanine transaminase (ALT) (Fuji DRI Chem 3500, Tokyo, Japan) were measured. Rat ELISA kits for adiponectin (AdipoGen Inc, Seoul, Korea) and monocyte chemo-attractant protein-1 (MCP-1; Invitrogen) were used.

\section{Intrahepatic triglyceride content}

Frozen rat liver tissues were homogenized in $1 \mathrm{ml}$ of deionized water before adding $5 \mathrm{ml}$ of chloroform and methanol and were centrifuged at $4^{\circ} \mathrm{C}$ and $35,000 \mathrm{rpm}$ for 10 $\min$. The lower phase was transferred to a clean tube and $250 \mu \mathrm{l}$ of $1 \%$ Triton X-100 in chloroform was added. After vacuum drying to remove the chloroform, triglyceride level was measured using a kit (Cayman Chemical Company, Ann Arbor, MI).

\section{Real time RT-PCR}

Total RNA was isolated from 3T3-L1 adipocytes, HepG2 cells and liver tissues using TRIzol reagent (Invitrogen) as described by the manufacturer. Complementary DNA (cDNA) was synthesized from $1 \mu \mathrm{g}$ of RNA using a M-MLV reverse transcription system (Promega, Madison, WI). Real time RT-PCR was performed using a SYBR Green RT-PCR kit (Qiagen, Valencia, CA) and measured with a Roter-Gene RG-3000 cycler (Corbett Research, Mortlake, Australia). Relative gene expression for MCP-1, tumor necrosis factor- $\alpha$ (TNF- $\alpha$ ), PGC- $\alpha$, CPT-1, and nuclear factor-kappa $\mathrm{B}(\mathrm{NF}-\kappa \mathrm{B})$ was analyzed by the $2^{-\Delta \Delta \mathrm{Ct}}$ method, normalizing to GAPDH gene expression. Primer pairs are presented in Supplemental Data Table S1.

\section{Statistical analyses}

All data are presented as means \pm S.D. Statistical analysis was carried out by ANOVA, followed by Tukey test for multiple comparisons using the SPSS 17.0 program. Differences were considered significant at $P<0.05$.

\section{Supplemental data}

Supplemental data include a table and can be found with this article online at http://e-emm.or.kr/article/article_files/ SP-44-10-02.pdf.

\section{Acknowledgements}

This study was supported by a research grant from Yonsei University Wonju College of Medicine (YUWCM 2010). This research was supported by Basic Science Research Program (2009-0064302) and Mid-career Researcher Program (2010-0005071) through the National Research Foundation of Korea (NRF) grant funded by the Ministry of Education, Science and Technology. 


\section{References}

Barish GD, Atkins AR, Downes M, Olson P, Chong LW, Nelson M, Zou Y, Hwang HS, Kang HJ, Curtiss L, Evans RM, Lee $\mathrm{CH}$. PPARdelta regulates multiple proinflammatory pathways to suppress atherosclerosis. Proc Nati Acad Sci USA 2008;105:4271-6

Bataller R, Brenner DA. Liver fibrosis. J Clin Invest 2005;115: 209-18

Berger JP, Akiyama TE, Meinke PT. PPARs: therapeutic targets for metabolic disease. Trends Pharmacol Sci 2005; 26:244-51

Bugianesi E, McCullough AJ, Marchesini G. Insulin resistance: a metabolic pathway to chronic liver disease. Hepatology 2005;42:987-1000

Evans RM, Barish GD, Wang YX. PPARs and the complex journey to obesity. Nat Med 2004;10:355-61

Fan Y, Wang Y, Tang Z, Zhang H, Qin X, Zhu Y, Guan Y, Wang $X$, Staels $B$, Chien $S$, Wang $N$. Suppression of proinflammatory adhesion molecules by PPAR-delta in human vascular endothelial cells. Arterioscler Thromb Vasc Biol 2008;28:315-21

Fasshauer M, Klein J, Kralisch S, Klier M, Lossner U, Bluher $\mathrm{M}$, Paschke R. Monocyte chemoattractant protein 1 expression is stimulated by growth hormone and interleukin-6 in 3T3-L1 adipocytes. Biochem Biophys Res Commun 2004;317:598-604

Gao D, Nong S, Huang X, Lu Y, Zhao H, Lin Y, Man Y, Wang $\mathrm{S}$, Yang J, Li J. The effects of palmitate on hepatic insulin resistance are mediated by NADPH Oxidase 3-derived reactive oxygen species through JNK and p38MAPK pathways. J Biol Chem 2010;285:29965-73

Jeong IK, Oh DH, Park SJ, Kang JH, Kim S, Lee MS, Kim MJ, Hwang YC, Ahn KJ, Chung HY, Chae MK, Yoo HJ. Inhibition of NF-KB prevents high glucose-induced proliferation and plasminogen activator inhibitor-1 expression in vascular smooth muscle cells. Exp Mol Med 2011;43:684-92

Joshi-Barve S, Barve SS, Amancherla K, Gobejishvili L, Hill $D$, Cave M, Hote P, McClain CJ. Palmitic acid induces production of proinflammatory cytokine interleukin-8 from hepatocytes. Hepatology 2007;46:823-30

Kahri J, Vuorinen-Markkola H, Tilly-Kiesi M, Lahdenpera S, Taskinen MR. Effect of gemfibrozil on high density lipoprotein subspecies in non-insulin dependent diabetes mellitus. Relations to lipolytic enzymes and to the cholesteryl ester transfer protein activity. Atherosclerosis 1993;102:79-89

Kliewer SA, Lehmann JM, Willson TM. Orphan nuclear receptors: shifting endocrinology into reverse. Science 1999;284:757-60

Koo SH, Satoh H, Herzig S, Lee CH, Hedrick S, Kulkarni R, Evans RM, Olefsky J, Montminy M. PGC-1 promotes insulin resistance in liver through PPAR-alpha-dependent induction of TRB-3. Nat Med 2004;10:530-4

Lawson JA, Fisher MA, Simmons CA, Farhood A, Jaeschke $\mathrm{H}$. Parenchymal cell apoptosis as a signal for sinusoidal sequestration and transendothelial migration of neutrophils in murine models of endotoxin and Fas-antibody-induced liver injury. Hepatology 1998;28:761-7

Liang $\mathrm{H}$, Ward WF. PGC-1alpha: a key regulator of energy metabolism. Adv Physiol Educ 2006;30:145-51

Lin J, Wu H, Tarr PT, Zhang CY, Wu Z, Boss O, Michael LF, Puigserver $\mathrm{P}$, Isotani E, Olson EN, Lowell BB, Bassel-Duby $\mathrm{R}$, Spiegelman BM. Transcriptional co-activator PGC-1 alpha drives the formation of slow-twitch muscle fibres. Nature 2002;418:797-801

Marchesini G, Bugianesi E, Forlani G, Cerrelli F, Lenzi M, Manini R, Natale S, Vanni E, Villanova N, Melchionda N, Rizzetto M. Nonalcoholic fatty liver, steatohepatitis, and the metabolic syndrome. Hepatology 2003;37:917-23

McCullough AJ. Pathophysiology of nonalcoholic steatohepatitis. J Clin Gastroenterol 2006;40:S17-29

Michael LF, Wu Z, Cheatham RB, Puigserver P, Adelmant G, Lehman JJ, Kelly DP, Spiegelman BM. Restoration of insulin-sensitive glucose transporter (GLUT4) gene expression in muscle cells by the transcriptional coactivator PGC-1. Proc Natl Acad Sci USA 2001;98:3820-5

Miyazaki Y, Mahankali A, Matsuda M, Glass L, Mahankali S, Ferrannini E, Cusi K, Mandarino LJ, DeFronzo RA. Improved glycemic control and enhanced insulin sensitivity in type 2 diabetic subjects treated with pioglitazone. Diabetes Care 2001;24:710-9

Nolan JJ, Ludvik B, Beerdsen P, Joyce M, Olefsky J. Improvement in glucose tolerance and insulin resistance in obese subjects treated with troglitazone. N Engl J Med 1994; 331:1188-93

Oliver WR Jr , Shenk JL, Snaith MR, Russell CS, Plunket KD, Bodkin NL, Lewis MC, Winegar DA, Sznaidman ML, Lambert MH, Xu HE, Sternbach DD, Kliewer SA, Hansen BC, Willson TM. A selective peroxisome proliferator-activated receptor delta agonist promotes reverse cholesterol transport. Proc Natl Acad Sci USA 2001;98:5306-11

Rodriguez-Calvo R, Serrano L, Coll T, Moullan N, Sanchez RM, Merlos M, Palomer X, Laguna JC, Michalik L, Wahli W, Vázquez-Carrera M. Activation of peroxisome proliferatoractivated receptor beta/delta inhibits lipopolysaccharideinduced cytokine production in adipocytes by lowering nuclear factor-kappaB activity via extracellular signalrelated kinase 1/2. Diabetes 2008;57:2149-57

Shoelson SE, Herrero L, Naaz A. Obesity, inflammation, and insulin resistance. Gastroenterology 2007;132:2169-80

Tanaka T, Yamamoto J, Iwasaki S, Asaba H, Hamura H, Ikeda Y, Watanabe M, Magoori K, loka RX, Tachibana K, Watanabe Y, Uchiyama Y, Sumi K, Iguchi H, Ito S, Doi T, Hamakubo T, Naito M, Auwerx J, Yanagisawa M, Kodama T, Sakai J. Activation of peroxisome proliferator-activated receptor delta induces fatty acid beta-oxidation in skeletal muscle and attenuates metabolic syndrome. Proc Natl Acad Sci USA 2003;100:15924-9

Tarantino G, Savastano S, Colao A. Hepatic steatosis, lowgrade chronic inflammation and hormone/growth factor/ adipokine imbalance. World J Gastroenterol 2010;16: 4773-83

Wellen KE, Hotamisligil GS. Inflammation, stress, and diabetes. J Clin Invest 2005;115:1111-9 